\title{
Probability Distribution Function of the Diquark Condensate in Two Colours QCD
}

\author{
R. Aloisio ${ }^{a, d}$, V. Azcoiti ${ }^{b}$, G. Di Carlo ${ }^{c, d}$, A. Galante $^{a, d}$ and A.F. Grillo ${ }^{d}$ \\ a Dipartimento di Fisica dell'Università di L'Aquila, 67100 L'Aquila, (Italy). \\ ${ }^{b}$ Departamento de Física Teórica, Facultad de Ciencias, Universidad de Zaragoza, \\ 50009 Zaragoza (Spain). \\ ${ }^{c}$ Istituto Nazionale di Fisica Nucleare, Laboratori Nazionali di Frascati, \\ P.O.B. 13 - 00044 Frascati, (Italy). \\ ${ }^{d}$ Istituto Nazionale di Fisica Nucleare, Laboratori Nazionali del Gran Sasso, \\ 67010 Assergi (L'Aquila), (Italy).
}

\begin{abstract}
We consider diquark condensation in finite density lattice $\mathrm{SU}(2)$. We first present an extension of Vafa-Witten result, on spontaneous breaking of vectorlike global symmetries, that allows us to formulate a no-go theorem for diquark condensation in a region of the chemical potential-mass parameter space. We then describe a new technique to calculate diquark condensation at any number of flavours directly at zero external source without using any potentially dangerous extrapolation procedure. We apply it to the strong coupling limit and find compelling evidences for a second order phase transition, where a diquark condensate appears, as well as quantitative agreement between lattice results and low-energy effective Lagrangian calculations.
\end{abstract}




\section{Introduction}

Recently the expected scenario for the phase diagram of QCD in the chemical potential-temperature plane has changed: besides the hadronic and quarkgluon plasma phases, the existence of a new state of matter has been proposed by several groups [1]. This new phase is characteristic of the high density, low temperature regime: the asymptotic freedom of QCD and the known instability of large Fermi spheres, in presence of (whatever weak) attractive forces, results in a pairing of quarks with momenta near the Fermi surface (in analogy with the Cooper pairing in solid state systems at low temperature). A condensation of quark pairs should be the distinctive signal of the new phase and has been indeed predicted using simplified phenomenological models of QCD.

Unfortunately the lattice approach, the most powerful tool to perform first principles, non perturbative studies, is affected in the case of finite density QCD by the well known sign problem that has prevented until now any step towards the understanding of this new phase. This is not the case for the $\mathrm{SU}(2)$ theory where the fermions are in the pseudo-real representation and finite density numerical simulations are feasible.

In this paper we present a detailed study of diquark condensation for the unquenched two colours model. This work follows a previous one where we considered chiral and diquark susceptibilities and found strong evidence for a phase transition separating the ordinary low density phase from a high density one where the chiral condensate vanishes and the baryon number symmetry is broken [2]. In the present work we mainly focus on an approach, based on the analysis of the probability distribution function (p.d.f.) of the order parameter for Grassmann fields [5], to compute the value of the order parameter directly at zero external source without using any potentially dangerous extrapolation procedure, mandatory in the standard approach.

This technique has also another relevant feature. Since configurations are generated directly at zero diquark source, the sign ambiguity in the definition of the fermionic partition function is avoided. It turns out that we are not restricted to $n_{f}=8$ but any number of flavours can be in principle simulated.

The study of diquark condensation in two colours QCD is an interesting topic by itself and we can hope to use some of the results to get insights about the three colours case. We are interested in the similarities between 
the phase diagram of $\mathrm{SU}(3)$ and $\mathrm{SU}(2)$, however we have to be aware of the differences between the two models:

- The quark-quark condensate $\langle q q\rangle$ is coloured for the $N_{c}=3$ theory and colourless for $N_{c}=2$. This implies that in the $\mathrm{SU}(3)$ case the diquark condensation has to be interpreted as a Higgs-like mechanism leading to the breaking of the local colour symmetry, while in the $\mathrm{SU}(2)$ case we have spontaneous symmetry breaking (SSB) of the $U_{B}(1)$ global symmetry associated with conservation of baryon number.

- The zero temperature critical chemical potential, related to the mass of the lightest baryonic state, is different in the two cases: it is expected to be $1 / 3$ of the nucleon mass for $\mathrm{SU}(3)$ and $1 / 2$ of the pion mass for $\mathrm{SU}(2)$ (i.e. zero at vanishing quark mass). For $N_{c}=2$, in order to have a phase diagram similar to that of $\mathrm{SU}(3)$, it is appropriate to consider a non zero bare quark mass.

- The lightest baryonic state is a fermion for the tree colours model and a boson for the two colours one.

Our results can be used to do a highly non trivial, quantitative check of the continuum predictions for the $\mathrm{SU}(2)$ theory, as for instance those from low energy effective Lagrangian calculations [3].

In the next section we present an extension, to $\mathrm{SU}(2)$ gauge theory at finite chemical potential, of the Vafa-Witten theorem on the impossibility to break spontaneously baryon number conservation in a vector-like theory [四, finding an analytical bound, useful to check approximated models. In section III we give a review of the p.d.f. formalism for Grassmann degrees of freedom, emphasizing on the details of its application to the analysis of the order parameter for the $\mathrm{U}(1)$ symmetry associated to baryon number conservation. In section IV we present some details of the numerical algorithm. The last section is devoted to the presentation and discussion of numerical results.

\section{The Vafa-Witten theorem at finite $\mu$}

In their original paper [4] Vafa and Witten proved that the two point correlation function of any operator with non zero isospin or baryon number (or any other conserved quantity carried by fermions but not by gauge bosons) 
falls off exponentially at large distances. From this result they concluded that any order parameter for such vector-like global symmetries is zero and that massless bound states can only be built by zero bare mass constituents.

The two main ingredients that are necessary to the proof are (i) the antihermiticity of the massless Dirac operator (to bound the fermion propagator for a given gauge background) and (ii) the positivity of the integration measure (used to extend the above bound to the averaged correlation function). These conditions limit the applicability of the theorem to vector-like theories (Yukawa coupling generally invalidates the argument) at $\theta=0$ and zero chemical potential.

We are here interested to the case in which the CP-violating vacuum angle vanishes but the chemical potential $\mu$ is non zero. While for $\mathrm{SU}(3)$ this breaks both conditions, for two colours QCD only condition (i) is not fulfilled. We will show how the theorem can be extended to the latter case for a range of values of $\mu$ that depends on the bare quark mass. Such new version of the theorem will allow us to exclude spontaneous breaking of baryon number (i.e. the formation of a diquark condensate that breaks baryon number conservation) for some region of the parameter space.

In this work we consider the theory regularized on the lattice. In such a case there is no need to consider smeared operators as in the original paper and we have to prove a uniform upper bound for the fermion propagator

$$
\left|\left\langle x\left|\Delta^{-1}(\mu)\right| 0\right\rangle\right| \leq \alpha \exp (-\beta|x|)
$$

where $\Delta(\mu)$ is the Dirac operator on the lattice in presence of a chemical potential term and $\alpha, \beta$ are constants. $\Delta(\mu)$ can be written as the sum of the Dirac operator at zero chemical potential $(\Delta(0)=i \Lambda+m)$ plus a term containing all the $\mu$ dependence: $\Delta(\mu)=\Delta(0)+\epsilon(\mu)$. The matrix $\epsilon(\mu)=\left(e^{\mu}-1\right) G+\left(1-e^{-\mu}\right) G^{\dagger}$ contains only the forward $(G)$ and backward $\left(G^{\dagger}\right)$ temporal links and is bounded: $\|\epsilon(\mu)\| \leq 2 \sinh \mu$. Expanding the inverse of the Dirac operator円

$$
\begin{aligned}
\Delta^{-1}(\mu) & =\left(I+\Delta^{-1}(0) \epsilon(\mu)\right)^{-1} \Delta^{-1}(0) \\
& =\Delta^{-1}(0)-\Delta^{-1}(0) \epsilon(\mu) \Delta^{-1}(0)+\Delta^{-1}(0) \epsilon(\mu) \Delta^{-1}(0) \epsilon(\mu) \Delta^{-1}(0) \\
& +\cdots
\end{aligned}
$$

\footnotetext{
${ }^{1}$ The expansion is valid if $\left\|\Delta^{-1}(0) \epsilon(\mu)\right\|<1$. Using $\left\|\Delta^{-1}(0)\right\| \leq m^{-1}$ and the bound on $\epsilon(\mu)$ the above condition is fulfilled if $m>2 \sinh \mu$. At the end we will check that our result is inside this region.
} 
and using an integral representation for $\Delta^{-1}(0)$ we get the following series expansion for the fermion propagator:

$$
\begin{aligned}
& \left\langle x\left|\Delta^{-1}(\mu)\right| 0\right\rangle=\int_{0}^{\infty} d \tau_{0} e^{-m \tau_{0}}\left\langle x\left|e^{-i \tau_{0} \Lambda}\right| 0\right\rangle \\
& \quad+\iint_{0}^{\infty} d \tau_{0} d \tau_{1} e^{-m\left(\tau_{0}+\tau_{1}\right)}\left\langle x\left|e^{-i \tau_{0} \Lambda} \epsilon(\mu) e^{-i \tau_{1} \Lambda}\right| 0\right\rangle \\
& \quad-\iiint_{0}^{\infty} d \tau_{0} d \tau_{1} d \tau_{2} e^{-m\left(\tau_{0}+\tau_{1}+\tau_{2}\right)}\left\langle x\left|e^{-i \tau_{0} \Lambda} \epsilon(\mu) e^{-i \tau_{1} \Lambda} \epsilon(\mu) e^{-i \tau_{2} \Lambda}\right| 0\right\rangle \\
& \quad+\cdots
\end{aligned}
$$

The expectation values in the r.h.s. of (3) can be bounded depending on the value of the integration variables: since

$$
P_{n}(x, 0)=\left|\left\langle x\left|e^{-i \tau_{0} \Lambda} \epsilon(\mu) \ldots e^{-i \tau_{n} \Lambda}\right| 0\right\rangle\right| \leq\|\epsilon(\mu)\|^{n} \sum_{k \geq x} \frac{\|\Lambda\|^{k}}{k !}\left(\tau_{0}+\ldots+\tau_{n}\right)^{k}
$$

we get the relations

$$
P_{n}(x, 0) \leq \begin{cases}\|\epsilon(\mu)\|^{n} e^{-\|\Lambda\| x} & \tau_{0}+\ldots \tau_{n} \leq x e^{-2\|\Lambda\|} \\ \|\epsilon(\mu)\|^{n} & \text { otherwise }\end{cases}
$$

If we take the modulus of expression (3) and use relations (14) we get

$$
\left|\left\langle x\left|\Delta^{-1}(\mu)\right| 0\right\rangle\right| \leq \sum_{n} q_{n}+\sum r_{n}
$$

where

$$
\begin{aligned}
q_{n} & \leq \frac{\|\epsilon(\mu)\|^{n}}{m^{n+1}} e^{-\|\Lambda\| x} \\
r_{n} & \leq\|\epsilon(\mu)\|^{n} \int_{D_{n}} d \tau_{0} \ldots d \tau_{n} e^{-m\left(\tau_{0}+\tau_{1}+\ldots \tau_{n}\right)}
\end{aligned}
$$

and $D_{n}$ is the set of points that satisfy the condition $\tau_{0}+\ldots \tau_{n} \geq x \exp (-2\|\Lambda\|)$ Clearly $\sum q_{n}$ is bounded by a geometric series

$$
\sum q_{n} \leq e^{-\|\Lambda\| x} \frac{1}{m-\|\epsilon(\mu)\|} \quad \text { if } \quad\|\epsilon(\mu)\|<m
$$

We have to find an (exponential) bound for the other term in the r.h.s. of (5). Since

$$
I_{0}=\int_{D_{0}} d \tau_{0} e^{-m \tau_{0}}=\frac{1}{m} e^{-m x e^{-2\|\Lambda\|}}
$$


it is possible to calculate

$$
I_{n}=\int_{D_{n}} d \tau_{0} \ldots \tau_{n} e^{-m\left(\tau_{0}+\ldots \tau_{n}\right)}
$$

using the change of variables

$$
\begin{aligned}
\tau_{0}+\tau_{1}+\ldots \tau_{n} & =t_{0} \\
\tau_{1}+\ldots \tau_{n} & =t_{1} \\
& \vdots \\
\tau_{n} & =t_{n}
\end{aligned}
$$

It is easy to check that

$$
I_{n}=\frac{(-1)^{n}}{n !} \frac{d^{n} I_{0}}{d m^{n}}
$$

and finally

$$
\sum_{n} r_{n} \leq \sum_{k=0}^{\infty}(-1)^{k} \frac{\|\epsilon(\mu)\|^{k}}{k !} \frac{d^{n} I_{0}}{d m^{n}}
$$

Note that $I_{0}$ is an analytic function of $m$ for $m \neq 0$ so the r.h.s. of (7) is equal to the Taylor expansion of $I_{0}(m-\|\epsilon(\mu)\|)$ around $I_{0}(m)$ provided $m>\|\epsilon(\mu)\|$.

Putting together the last result with (6) and the condition for the Taylor expansion in (2) we get the final bound

$$
\left|\left\langle x\left|\Delta^{-1}(\mu)\right| 0\right\rangle\right| \leq \frac{1}{m-\|\epsilon(\mu)\|}\left(e^{-\|\Lambda\| x}+e^{-(m-\|\epsilon(\mu)\|) x \exp (-2\|\Lambda\|)}\right)
$$

valid for any $m>\|\epsilon(\mu)\|$. Since $\|\epsilon(\mu)\| \leq 2 \sinh \mu$ we conclude that the fermionic correlation function decays exponentially at least for any

$$
m>2 \sinh \mu
$$

Note that in the $\mu \rightarrow 0$ limit we recover the original result of Vafa and Witten i.e. the propagator goes to zero exponentially for any nonzero mass.

The formation of a diquark condensate, as follows from (9), is excluded for $\mu<\tilde{\mu}=\sinh ^{-1}(m / 2)$. Near the chiral limit, $\tilde{\mu}$ is smaller than half the pion mass so condition (9) is fulfilled for the expected critical point $\mu_{c}=m_{\pi} / 2$. 
Our result is interesting because it poses a rigorous limit to diquark condensation and this limit can be used as a check to phenomenological approximations to the model. It also shows how the Vafa-Witten theorem on spontaneous breaking of vector-like global symmetries can be extended, for some region of the parameter space, to a theory at non zero chemical potential.

\section{The probability distribution function of the diquark condensate}

The use of the p.d.f. to analyze the spontaneous symmetry breaking in spin systems or Quantum Field Theories with bosonic degrees of freedom is a standard procedure. Less standard is its application to QFT with Grassmann fields where the fermionic degrees of freedom have to be integrated analytically.

The version of this method we use has been developed to extract the chiral condensate in the chiral limit from simulations of QFT with fermions [5], and will be used to study the vacuum structure of two colours QCD at non zero density and specifically to extract the diquark condensate at $j=0$. We refer to the original paper [5] for a full description of the p.d.f. technique and present a brief introduction focusing on the peculiarities of the diquark condensate case.

In analogy with the study of chiral symmetry, we can construct a two component diquark condensate vector $\left(\left\langle\psi \tau_{2} \psi+\bar{\psi} \tau_{2} \bar{\psi}\right\rangle, i\left\langle\psi \tau_{2} \psi-\bar{\psi} \tau_{2} \bar{\psi}\right\rangle\right)$ which transforms under $\mathrm{U}(1)_{B}$ as a vector under rotations in the plane. Therefore we can take any of these two diquark condensates as order parameter $c$ for the $\mathrm{U}(1)_{B}$ symmetry associated to baryon number conservation. In the following we will use the first component. Then let $\alpha$ be an index which characterizes all possible (degenerate) vacuum states and $w_{\alpha}$ the probability to get the vacuum state $\alpha$ when choosing randomly an equilibrium state. If $c_{\alpha}$ is the value taken by the order parameter in the $\alpha$ state, we can write

$$
c_{\alpha}=\left\langle\frac{1}{V} \sum_{x}\left(\psi \tau_{2} \psi(x)+\bar{\psi} \tau_{2} \bar{\psi}(x)\right)\right\rangle_{\alpha}
$$

where $V$ is the lattice volume and the sum is over all lattice points. $P(c)$, 
the p.d.f. of the diquark order parameter $c$, will be given by

$$
\begin{aligned}
P(c) & =\sum_{\alpha} w_{\alpha} \delta\left(c-c_{\alpha}\right) \\
& =\lim _{V \rightarrow \infty} \frac{1}{\mathcal{Z}} \int[d U][d \bar{\psi}][d \psi] e^{-S_{G}(U)+\bar{\psi} \Delta \psi} \delta\left(\frac{1}{V} \sum_{x} \psi \tau_{2} \psi(x)+\bar{\psi} \tau_{2} \bar{\psi}(x)-c\right)
\end{aligned}
$$

The main point is that while $P(c)$ is not directly accessible with a numerical simulation its Fourier transform

$$
P(q)=\int d c e^{i q c} P(c)
$$

can be easily computed. Inserting in (11) the definition of $P(c)$ and using an integral representation for the $\delta$-function, we can compute the integral over the Grassmann variables:

$$
\int[d \bar{\psi}][d \psi] e^{\bar{\psi} \Delta \psi+i q / V\left(\psi \tau_{2} \psi+\bar{\psi} \tau_{2} \bar{\psi}\right)}=\operatorname{PfB}\left(\frac{i q}{V}\right)
$$

where we have defined

$$
B(j)=\left(\begin{array}{cc}
j & \frac{1}{2} \Delta \tau_{2} \\
-\frac{1}{2} \Delta^{T} \tau_{2} & j
\end{array}\right) .
$$

and $\Delta$ is the usual lattice Dirac operator (it contains the mass and $\mu$ dependence).

After some algebra we obtain:

$$
P_{V}(q)=\frac{1}{\mathcal{Z}} \int[d U] e^{-S_{G}(U)} \frac{\operatorname{Pf} B\left(\frac{i q}{V}\right)}{\operatorname{det} \Delta}[\operatorname{det} \Delta]^{\frac{N_{f}}{4}}
$$

where $P_{V}(q)$ is the Fourier transformed p.d.f. of the diquark order parameter at zero external source and finite volume, $\mathcal{Z}$ is the standard partition function.

To take advantage of (14) we should be able to compute correctly the Pfaffian involved. This indeed turns out to be easy. The only ambiguity is related to the sign of $\operatorname{Pf} B\left(\frac{i q}{V}\right)= \pm \sqrt{\operatorname{det} B(i q / V)}$. In the next section we will analize some properties of the matrix $B$ and show how it is possible to compute $\operatorname{Pf} B(i q / V)$ once we have the eigenvalues of $B(0)$.

As we will discuss in the next section, in standard simulations it is practically impossible to determine the sign of the Pfaffians involved and one is 
forced to choose $N_{f}$ equal to a multiple of 8 . A remarkable property of our approach is that, since we are able to compute $\operatorname{Pf} B(i q / V)$, any value of $N_{f}$ can be considered in the simulations.

Once we have the $P_{V}(q)$ and hence, by simple Fourier transform, the p.d.f. of the order parameter we need to extract the correct value for the order parameter. To do that we first have to recall that diquark condensate is the order parameter of the $U_{B}(1)$ symmetry associated with baryon number conservation and that $\left\langle\psi \tau_{2} \psi+\bar{\psi} \tau_{2} \bar{\psi}\right\rangle$ and $i\left\langle\psi \tau_{2} \psi-\bar{\psi} \tau_{2} \bar{\psi}\right\rangle$ are the components of a vector (in a plane) which rotates by an angle $2 \alpha$ when we do a global phase transformation of parameter $\alpha$ on the fermionic fields. Therefore, if $c_{0}$ is the vacuum expectation value of the diquark condensate (in the infinite volume limit) corresponding to the $\alpha$-vacuum selected by a diquark source term after taking the zero source limit, $P(c)$ can be computed as

$$
P(c)=\frac{1}{2 \pi} \int d \alpha \delta\left(c-c_{0} \cos (2 \alpha)\right)
$$

which gives $P(c)=1 /\left(\pi\left(c_{0}^{2}-c^{2}\right)^{1 / 2}\right)$ for $-c_{0} \leq c \leq c_{0}$ and $P(c)=0$ otherwise[0]. In the symmetric phase $c_{0}=0$ and $P(c)$ reduces to a $\delta$-function in the origin.

The above results are valid in the thermodynamic limit while, at finite volume, the non analyticities of the p.d.f. are absent. Even without performing a detailed finite size scaling analysis, we expect, for the finite volume p.d.f. $P_{V}(c)$, a function peaked in the origin in the symmetric phase and peaked at some non zero value in the broken phase. This is indeed the behaviour we can observe in fig. 1 where the the p.d.f. of the smallest volume simulated (see Section 5) is reported at different values of $\mu$. It is clear that, increasing the chemical potential, the vacuum starts to be degenerate signalling a spontaneous breaking of the baryon number conservation.

We can also compare $P_{V}(c)$ for two lattice volumes in the symmetric and broken phase. This is done in fig. 2 where we see clearly as, increasing the volume, the peak of the p.d.f. becomes sharper. To determine the value of the diquark condensate we used the position of the peak: a definition that clearly converges to the correct value in the thermodynamic limit.

From fig. 2 we see also that data of the larger volume are more noisy (indeed we also get negative values for the p.d.f. in the broken phase). To have an estimate of the errors we calculated several distribution functions 
for the $6^{4}$ lattice using the jacknife procedure. We saw that, except for the critical region, the position of the peak was very stable.

\section{Simulation scheme}

The standard way to study SSB is to introduce first an explicit symmetry breaking term in the action. If we do that for the diquark in the $\mathrm{SU}(2)$ model we have to add a term [6] $j\left(\psi \tau_{2} \psi+\bar{\psi} \tau_{2} \bar{\psi}\right)$ and, after integrating the Grassmann field, the fermionic contribution for $N_{f}=4$ quark flavours becomes proportional to the Pfaffian of a $4 \mathrm{~V} \times 4 \mathrm{~V}$ matrix [2]:

$$
\mathcal{Z}_{f e r m}(j)=\operatorname{Pf} B(j)= \pm \sqrt{\operatorname{det} B(j)}
$$

where $B(j)$ is defined in the previous section and $j$ is real.

Using the relation $\tau_{2} \Delta \tau_{2}=\Delta^{*}$ we can easily prove that $B(0)$ is antihermitian and $\operatorname{det} B(j) \geq 0$ for any $j$. It can also be shown that the eigenvalues of $B(0)$ are doubly degenerate and $B^{2}(0)$ is block diagonal with two hermitian blocks on the diagonal having the same eigenvalues. It follows that to compute $\operatorname{det} B(j)$ for any $j$ (i.e. to obtain all the eigenvalues of $B(0)$ ) it is sufficient to diagonalize only one block of $B^{2}(0)$ (reducing the problem to the diagonalization of a $2 \mathrm{~V} \times 2 \mathrm{~V}$ hermitian matrix) and then take the two (imaginary) square roots of its (real and negative) eigenvalues.

To avoid the sign ambiguity in (16) it is customary to consider a theory with $N_{f}=8$ quark flavours where the fermionic partition function becomes $\mathcal{Z}_{\text {ferm }}(j)=\operatorname{det} B(j)$. This limitation can be overcomeby exploiting our ability to work directly at zero diquark source. In the $j=0$ limit the sign ambiguity disappears and the Pfaffian is positive definite since $\operatorname{Pf} B(0) \equiv \operatorname{det} \Delta$ and the last quantity is real and positive for any value of $\mu$. Then we can easily consider any value of $N_{f}$ writing $\mathcal{Z}_{\text {ferm }}(j=0)=(\operatorname{det} B(0))^{N_{f} / 8}$.

If we are interested in the p.d.f. of the diquark order parameter at zero external source, the Pfaffian in (14) can also be easily computed. The non degenerate eigenvalues of $B(0)$ can be written as $\pm i \lambda_{n}(n=1, \cdots, V)$ with real and positive $\lambda_{n}$. Using the relation (16) we arrive at the following expression

$$
\operatorname{Pf} B\left(\frac{i q}{V}\right)= \pm \prod_{n=1}^{V}\left(\lambda_{n}^{2}-\frac{q^{2}}{V^{2}}\right)
$$


The sign ambiguity is solved noticing that (17) has to be positive at $j=0$. Increasing $q$, (17) changes sign each time $q / V$ is equal to one of the $\lambda_{n}$, except possibly in case of degeneracy in the eigenvalues of $B(0)$ resulting in the Pfaffian not crossing zero but tangent to the horizontal axis. This situation never occurred in our simulations.

The procedure we presented in previous section can be used for any value of the gauge coupling but we have studied the phase structure of the theory in the limit of infinite gauge coupling $(\beta=0)$. The main reason lies in the possibility to check our results with standard numerical simulations as well as analytical calculations. In this way we can restrict our efforts to the exploration of the phase space in the mass-chemical potential plane.

To simulate the $\beta=0$ limit of the theory we have measured fermionic observables on gauge configurations generated randomly, i.e. with only the Haar measure of the gauge group as a weight. This choice implies a Gaussian distribution of the plaquette energy around zero which, according to the results of Morrison and Hands [6], has a net overlap with the importance sample of gauge configurations at the values of $\mu$ and $m$ used in our calculations. The validity of this procedure has also been tested comparing different physical observables (number density and chiral condensate) with Hybrid Montecarlo results[7].

We have considered the theory in a $4^{4}$ and $6^{4}$ lattice diagonalizing 300 gauge configurations in the first lattice volume and 100 in the second one. As we pointed out in the introduction the simulations have been performed at non zero quark mass, in order to study a physical situation closer to SU(3). We choosed $m=0.025,0.05,0.20$ and values of the chemical potential ranging from $\mu=0$ to $\mu=1.0$. The values of the diquark condensate are determined from the position of the maximum of the probability distribution function and the errors have been determined using a jacknife procedure.

All numerical simulations have been performed on a cluster of PCs at the INFN Gran Sasso National Laboratory.

\section{Results and conclusions}

Here we present the results for the diquark condensate at $j=0$ as a function of the chemical potential.

Fig. 3 contains a comparison of $6^{4}$ and $4^{4}$ results for $N_{f}=1$ and $m=0.2$. 
We can clearly distinguish two symmetric phases separated by a broken one and two, possibly continuous, transition points. The high density symmetric phase has no physical relevance, being consequence of the saturation of all lattice sites with quarks. This phenomenum has nothing to do with continuum physics, being a pure lattice artifact. The physically interesting phase transition, i.e. the transition that has a continuum counterpart, is the first one [2] and it is the only one we will consider in the following.

With only two available volumes we do not have the possibility to perform a serious finite size scaling analisys. Anyway it is evident that increasing the lattice volume the behaviour near the (physical) critical point is more singular. A similar qualitative result holds for all the available masses and flavour numbers thus strongly supporting the picture of singular behaviour in the infinite volume limit.

We have also taken advantage of our simulation scheme considering different values of flavours. Increasing $N_{f}$ our operators become more noisy, especially near the transition point, but we can safely extend our calculations up to $N_{f}=4$. In fig. 4 we plot the diquark condensate for the largest mass and $N_{f}=1,2,4$. We see clearly that the three data set are almost coincident and we conclude that no dependence on $N_{f}$ is evident. Once again this result is valid also for the smaller masses.

The existence of spontaneous symmetry breaking with a non vanishing diquark condensate has already been predicted by Mean Field calculations [8] as well as numerical calculations [2, 9] and effective models [3]. We can use our relatively large data set to take a step forward and make a quantitative comparison with available analitical predictions.

In previous work we used low energy effective Lagrangian calculations to check $j \neq 0$ results finding remarkable agreement [2]. This motivated us to repeat the same procedure for our $j=0$ results. In this case the authors of [3] provide the following formula for the diquark condensate:

$$
\langle\psi \psi\rangle= \begin{cases}\langle\bar{\psi} \psi\rangle_{0} \sqrt{1-\left(\frac{m_{\pi}}{2 \mu}\right)^{4}} & \mu \geq m_{\pi} / 2 \\ 0 & \text { otherwise }\end{cases}
$$

where $\langle\bar{\psi} \psi\rangle_{0}, m_{\pi}$ are the chiral condensate and the pion mass at zero chemical potential.

We used (18) to fit our data for the larger lattice near the critical point: plotting $\mu^{4}\langle\psi \psi\rangle^{2}$ versus $\mu^{4}$ we expect a linear dependence. We used all the 
available masses and the $N_{f}=1$ results that have smaller errors. In fig. 5 the $m=0.025$ case is considered showing both the data points and the resulting linear fit. In all cases the $\chi^{2}$ is good (see table 1) and we conclude that (18) gives a good description of the numerical results.

In fig. 6 we have reported the diquark condensate as a function of $\mu$ for all the available masses. Clearly, away from the critical point, we can appreciate the dramatic effect of lattice discretization that prevents the diquark condensate to stay at the $\mu=0$ chiral condensate value (18).

In Table 1 we compare the results of the fits with the $\mu=0$ determinations of $\langle\bar{\psi} \psi\rangle$ and $m_{\pi} / 2$ performed on a $6^{3} \times 12$ lattice at $\beta=0$. The determination

Table 1: Parameters for the low energy effective Lagrangian predictions.

\begin{tabular}{|c|c|c|c|c|c|}
\hline$m$ & $\frac{1}{2} m_{\pi}$ (fit) & $\frac{1}{2} m_{\pi}$ & $\langle\bar{\psi} \psi\rangle_{0}$ (fit) & $\langle\bar{\psi} \psi\rangle_{0}$ & $\frac{\chi^{2}}{\text { d.o.f. }}$ \\
\hline 0.025 & $0.165(2)$ & $0.1696(11)$ & $1.325(10)$ & $1.31(2)$ & 1.1 \\
\hline 0.05 & $0.236(5)$ & $0.2405(9)$ & $1.29(2)$ & $1.29(2)$ & 2.2 \\
\hline 0.2 & $0.484(4)$ & $0.4841(7)$ & $1.438(13)$ & $1.23(2)$ & 3.3 \\
\hline
\end{tabular}

of the critical point and chiral condensate is in good agreement in the two data set, at least for the two smaller masses. Increasing $m$ the $\chi^{2}$ becomes larger and the determination of the chiral condensate is less accurate. This however is not surprising since we expect the validity range of the low energy Lagrangian prediction (18) to be restricted to the small quark mass region where the gap between the pion mass and the first non-Goldstone excitation is large [3].

What is more surprising is that, like in the $j \neq 0$ case [2], our $\beta=0$ calculations have a incredibly good agreement with a continuum (even if based on an effective model) prediction. Since the analytical predictions are, for the values of $\mu$ presented, well inside the validity region of the low energy approximation we can conclude that this gives indication of a very small dependence of lattice results on $\beta$.

It would be very interesting to test this prediction by performing finite coupling calculations. This can be done using our approach to extract directly the diquark order parameter at zero external source together with a HMC algorithm for generating configurations at $\beta \neq 0$. In this case we can compute $P_{V}(q)$ in (14) considering the ratio $\operatorname{PfB}(i q / V) / \operatorname{det} \Delta$ as an observ- 
able which, for each gauge configuration and $q<V$, is a number of order one (or smaller). This program has to face two difficulties: at zero temperature we need to increase our lattice temporal extent and correspondingly the computer time; on the other hand only unconclusive results are available for the $\mu=0$ thermodynamics of $\mathrm{SU}(2)$.

\section{Acknowledgments}

The authors thank M. P. Lombardo for providing the code necessary to extract the pion mass in $\mu=0$ simulations. This work has been partially supported by CICYT (Proyecto AEN97-1680) and by a INFN-CICYT collaboration. The Consorzio Ricerca Gran Sasso has provided part of the computer resources needed for this work. 


\section{References}

[1] M. Alford, K. Rajagopal and F. Wilczek, Phys. Lett. B422 (1998) 247; Nucl. Phys. B537 (1999) 443; R. Rapp, T. Schaefer, E.V. Shuryak and M. Velkovsky, Phys. Rev. Lett. 81 (1998) 53; T. Schafer and F. Wilczek, Phys. Rev. Lett. 82 (1999) 3956.

[2] R. Aloisio, V. Azcoiti, G. Di Carlo, A. Galante and A.F. Grillo, heplat/0009034, to appear on Phys. Lett. B.

[3] J.B. Kogut, M.A. Stephanov, D. Toublan, J.J.M. Verbaarschot and A. Zhitnitsky, hep-ph/0001171.

[4] C. Vafa and E. Witten, Nucl. Phys. B234 (1984) 173.

[5] V. Azcoiti, V. Laliena and X.Q. Luo, Phys. Lett. B354 (1995) 111.

[6] S. Morrison and S. Hands, in Strong and Electroweak Matter 98, Copenhagen, Dec. 1998, hep-lat/9902012.

[7] R. Aloisio, V. Azcoiti, G. Di Carlo, A. Galante and A.F. Grillo, Nucl. Phys. B564 (2000) 489.

[8] E. Dagotto, A. Moreo and U. Wolff, Phys. Lett. B186 (1987) 395.

[9] S. Hands and S. Morrison, in Understanding Deconfinement in QCD, Trento, March 1999, hep-lat/9905021. 


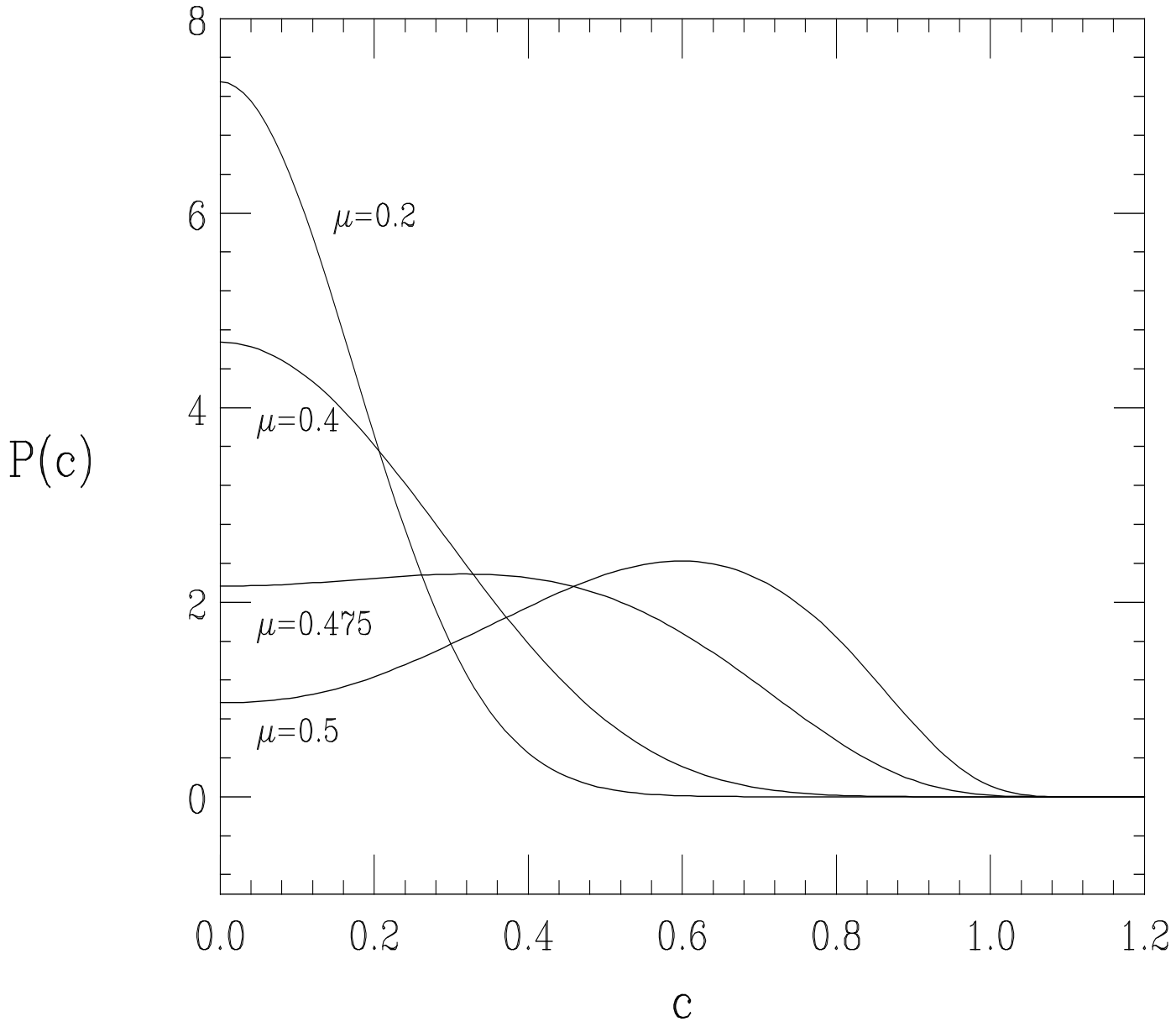

Figure 1: $P(c)$ for the $4^{4}$ lattice, $m=0.2$ at $\mu=0.2,0.4,0.475,0.5$ (from top to bottom). 

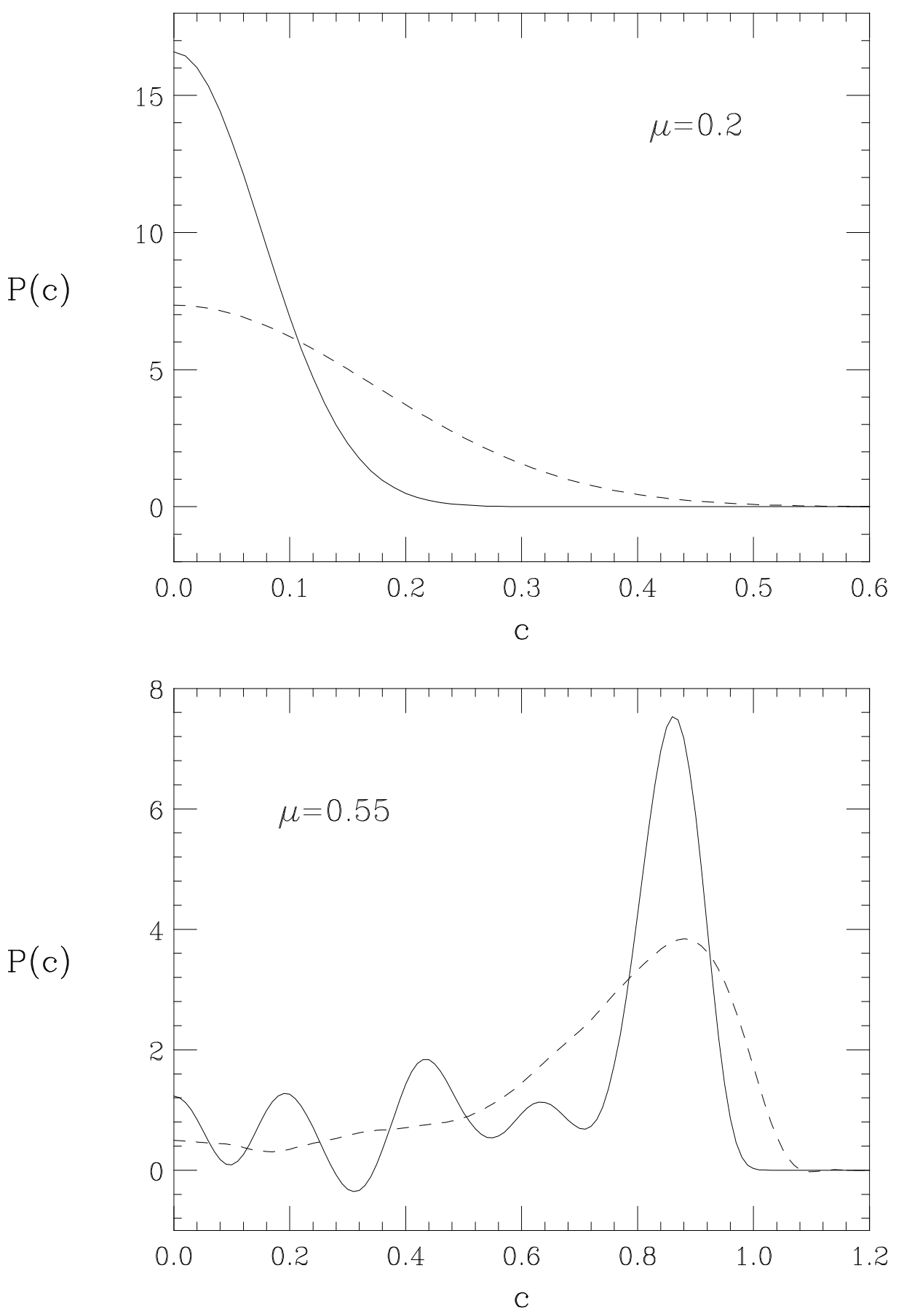

Figure 2: P.d.f. for $m=0.2: 4^{4}$ (dashed line) and $6^{4}$ (continuous line) in the symmetric (top) and broken (bottom) phase. 


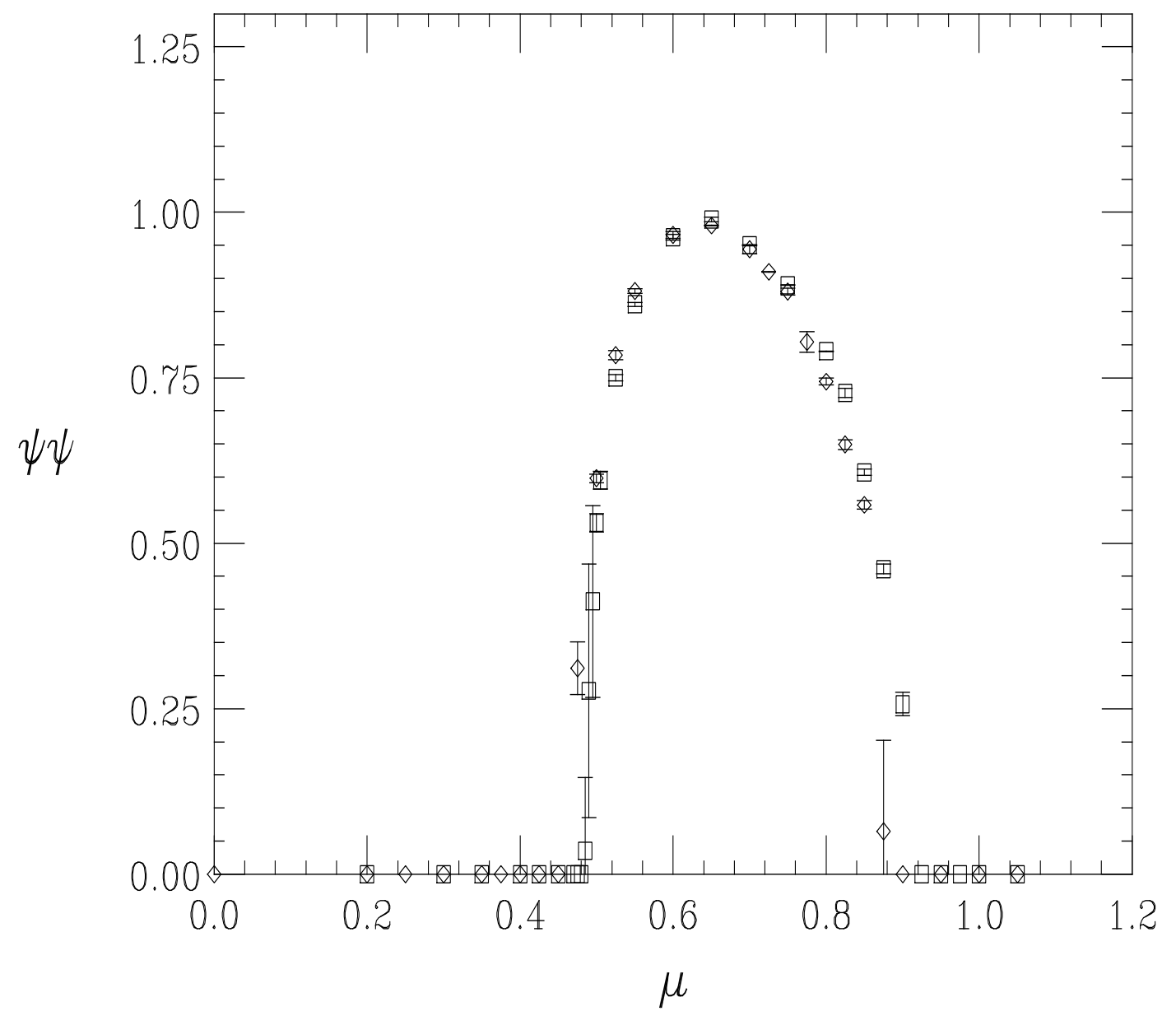

Figure 3: Diquark condensate for $4^{4}$ (diamonds) and $6^{4}$ (squares) lattices at $m=0.2, N_{f}=1$. 


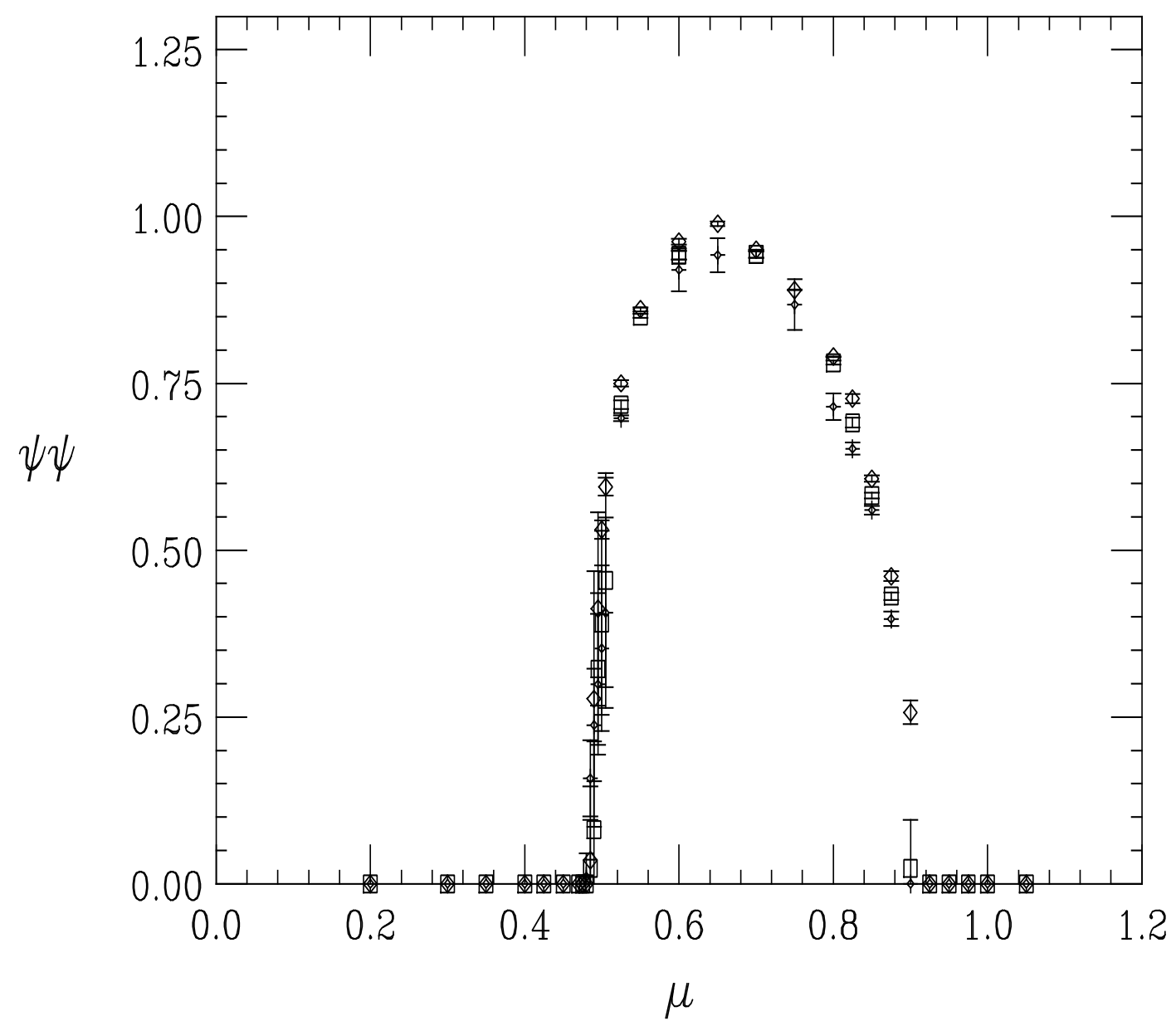

Figure 4: Diquark condensate for $N_{f}=1$ (diamonds), 2 (squares) and 4 (stars) in a $6^{4}$ lattice at $m=0.2$. 


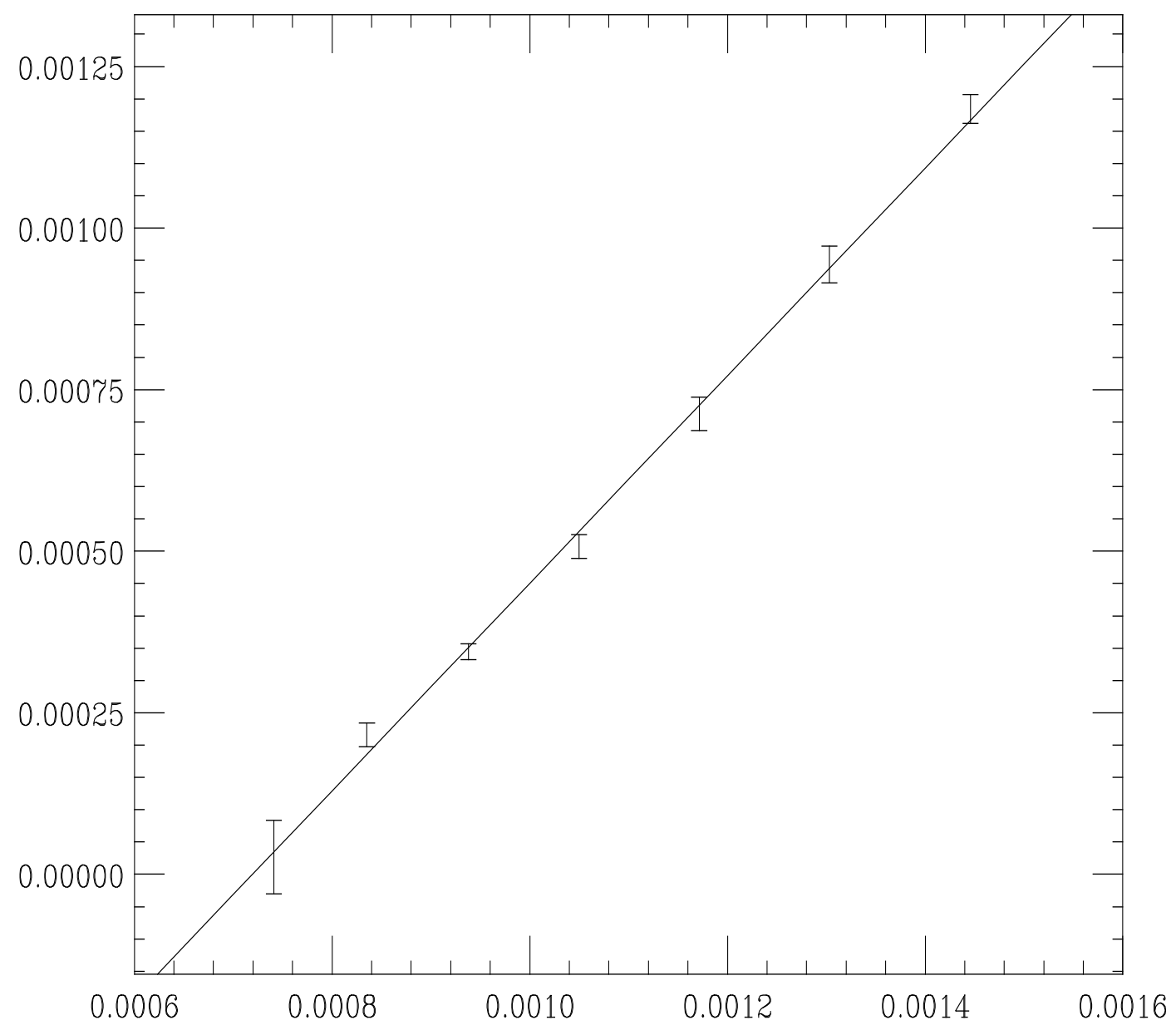

Figure 5: $\mu^{4}\langle\psi \psi\rangle^{2}$ vs $\mu^{4}$ for $6^{4}$ lattice, $m=0.025$ and linear best fit. 


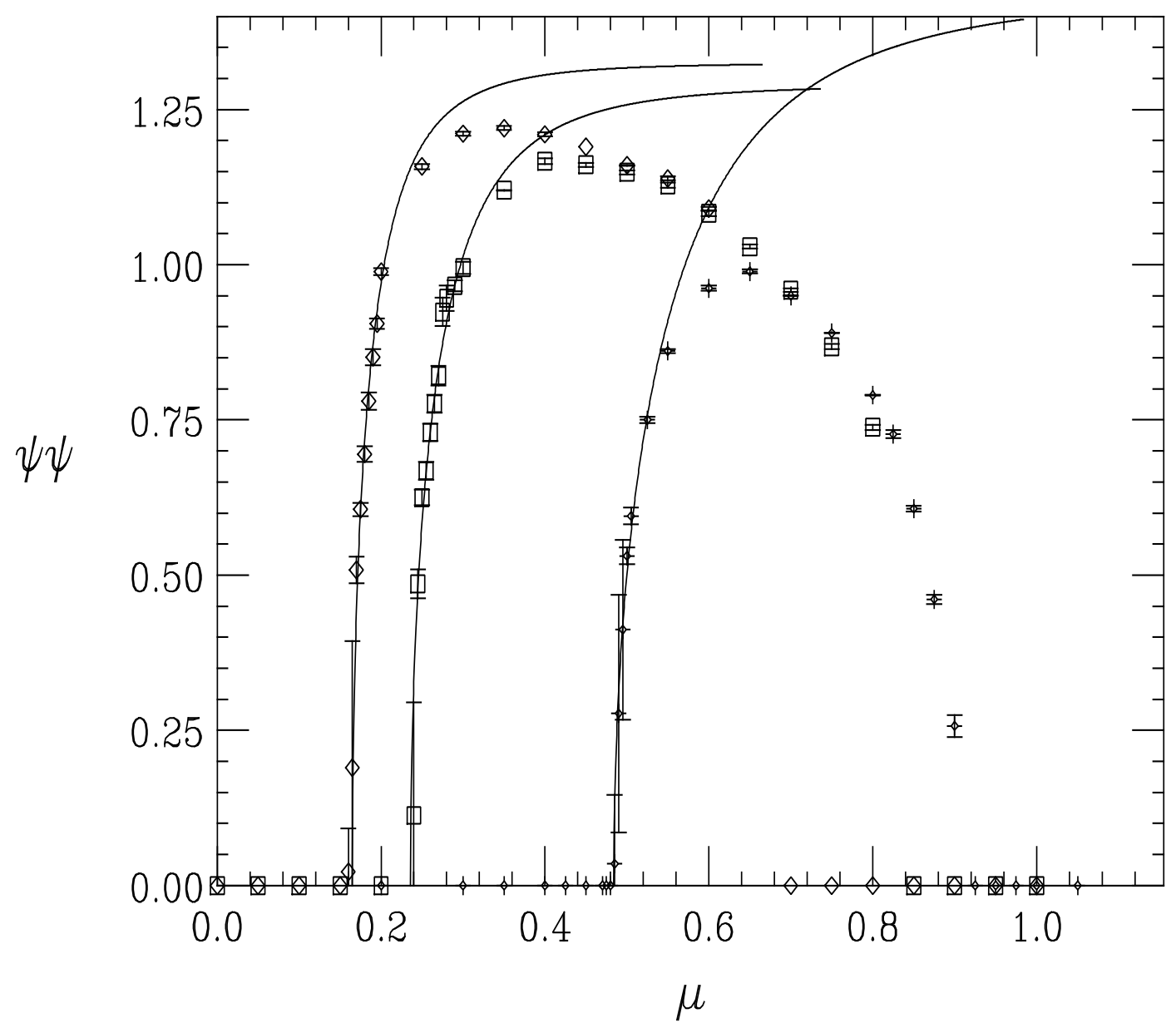

Figure 6: Diquark condensate for $6^{4}$ lattice, $N_{f}=1, m=0.025$ (diamonds), 0.05 (squares), 0.2 (stars) and corresponding fits using expression (18). 